\title{
Relativistic astrophysics and cosmology in Uzbekistan
}

\author{
Bobomurat J. Ahmedov ${ }^{1,2,3}$, Roustam M. Zalaletdinov ${ }^{1,4}$, \\ Zafar Ya. Turakulov ${ }^{1,2,3}$, Salakhutdin N. Nuritdinov ${ }^{2,3}$ \\ and Karomat T. Mirtadjieva ${ }^{2,3}$ \\ ${ }^{1}$ Institute of Nuclear Physics, Ulughbek, Tashkent 702132, Uzbekistan \\ ${ }^{2}$ Ulugh Beg Astronomical Institute, Astronomicheskaya 33, Tashkent 700052, Uzbekistan \\ ${ }^{3}$ National University of Uzbekistan Physics Faculty, Tashkent, 700174, Uzbekistan \\ ${ }^{4}$ Department of Mathematics, Statistics and Computer Science, St Francis Xavier University, \\ Antigonish, NS, Canada B2G 2W5 \\ email: ahmedov@astrin.uzsci.net,nurit@astrin.uzsci.net,mkt@astrin.uzsci.net
}

\begin{abstract}
The theoretical results obtained in Uzbekistan in the field of relativistic astrophysics and cosmology are presented. In particular electrostatic plasma modes along the open field lines of a rotating neutron star and Goldreich-Julian charge density in general relativity are analyzed for the rotating and oscillating magnetized neutron stars. The impact that stellar oscillations of different type (radial, toroidal and spheroidal ones) have on electric and magnetic fields external to a relativistic magnetized star has been investigated. A study of the dynamical evolution and the number of stellar encounters in globular clusters with a central black hole is presented. Perturbation features and instabilities of the large-scale oscillations on the background of the non-linearly pulsating isotropic and isotropic $\Omega$-models are studied. The non-stationary dispersion equation of the sectorial perturbations for the general case and the results of certain oscillation mode analysis are given. The model composed as the linear superposition of two other models was constructed and the stability of this model is studied. In a cosmological setting the theory of macroscopic gravity as a large-distance scale generalization of general relativity has been developed. Exact cosmological solutions to the equations of macroscopic gravity for a flat spatially homogeneous, isotropic space-time are found. The gravitational correlation terms in the averaged Einstein equations have the form of spatial curvature, dark matter and dark energy (cosmological constant) with particular equations of state for each correlation regime. Interpretation of these cosmological models to explain the observed large-scale structure of the accelerating Universe with a significant amount of the nonluminous (dark) matter is discussed.
\end{abstract}

Keywords. Theoretical astronomy, astronomy in Uzbekistan, rotating oscillating neutron stars, general relativity, dark matter, dark energy

\section{Introduction}

Theoretical Astrophysics is the subject which has got an essential development in Uzbekistan during the last decade, especially through the newly established collaborations with advanced western and eastern institutions. Our regional collaboration is supported by the Abdus Salam International Center for Theoretical Physics (ICTP, Trieste, Italy), Academy of Sciences for Developing World (TWAS, Trieste, Italy) and the Inter-University Centre for Astronomy and Astrophysics (IUCAA, Pune, India) in the framework of BIPTUN (Bangladesh - India - Pakistan - Turkey - Uzbekistan) Network on Relativistic Astrophysics and Cosmology. Another important scientific collaboration is with western partners, mainly at the International School for Advanced Studies (SISSA, Trieste, Italy), International Center for Relativistic Astrophysics (ICRA, Rome-Pescara, 
Italy), Dalhousie University (Halifax, Canada), St Francis Xavier University (Antigonish, Canada) and at the ICTP. Local scientific activity in Theoretical Astrophysics in Uzbekistan is partly supported through the Affiliation Scheme (ICAC-83) of the ICTP. Scientific collaboration of the Department of Galactic Astronomy and Cosmology in UBAI is with Kharkov State University (Ukraine), Sternberg Astronomical Institute (SAI) at Moscow State University, St Petersburg State University, the Astrophysical Institute (AIP, Potsdam) and international funding is mainly through Centre of Science and Technology in the Ukraine.

Research on Theoretical Astrophysics in Uzbekistan is concentrated in Ulugh Beg Astronomical Institute (UBAI) and Institute of Nuclear Physics (INP) of the Uzbekistan Academy of Sciences, and in the National University of Uzbekistan. UBAI is one of the oldest astronomical institutions of the Former Soviet Union. It was founded in 1873 and is the oldest one in the Central Asian area (where Uzbekistan lies), which has absolute maximum of clear sky time for the whole Eurasian continent. This makes the area particularly important for optical astronomical observations. As a result of the sitetesting expeditions organized by UBAI and SAI in the early 1970s, Maidanak mountain $(2700 \mathrm{~m})$ located $120 \mathrm{~km}$ south of the famous historical city of Samarkand, was selected for an observatory. In August 1996 a seeing monitoring at Mt Maidanak was started with Differential Image Motion Monitor of ESO, designed by M. Sarazin and used for Paranal and La Silla site-testing. After one year the results of the seeing measurements showed a very high quality of seeing conditions at Mt Maidanak.

Scientific projects in UBAI are related to the theoretical and observational research in galaxies, photometric observation of eclipsing binaries, observational studies of young stars, the study of solar activities etc., and two projects are related to theoretical astrophysics. These are F2.2.01 "Gravitational lenses and collapsing galaxies" and F2.2.06 "Dynamics of gravitating systems and electromagnetic fields around compact objects" where encounters in globular clusters and galaxies with a central black hole and electromagnetic fields and waves around magnetized neutron stars are investigated.

The Institute of Nuclear Physics (INP) in Tashkent is the biggest one in Central Asia. It was founded in year 1956. It has one project related to theoretical astrophysics: F2.1.09 entitled "Investigation of equations of gravitation and electrodynamics in relativistic astrophysics and cosmology".

Several scientists deliver lectures at the Astronomy Department of the National University of Uzbekistan, which was founded in the year 1918. The Astronomy Department of the University trains Bachelors, Masters and PhD students. The scientific activity of the Department members are in the fields of formation and evolution of elliptical galaxies, physics of globular clusters, dynamics of galaxy clusters, physics of quasars, and close binary systems. In particular the influence of the third star on close binary system is investigated there.

In this paper, the theoretical results obtained in Uzbekistan in the field of relativistic astrophysics of compact objects and relativistic cosmology will be presented. In particular electrostatic plasma modes along the open field lines of a rotating neutron star and Goldreich-Julian charge density in general relativity are analyzed for rotating and oscillating neutron stars. For a steady-state Goldreich-Julian charge density the usual plasma oscillation along the field lines are found; the plasma frequency resembles the gravitational redshift close to the Schwarzschild radius. The equations that describe the electromagnetic processes in a plasma surrounding a neutron star are obtained by using the general relativistic form of Maxwell's equations in a geometry of a slow-rotating gravitational object. A new mechanism of the generation of azimuthal current under the gravito-magnetic effect on radial current in a plasma around a neutron star is predicted. 
The impact that stellar oscillations have on electric and magnetic fields external to a relativistic magnetized star has been investigated. The solution of the general relativistic Maxwell equations both in the vicinity of the stellar surface and far from it has been found. The general relativistic energy loss through electromagnetic radiation for different types (radial, toroidal and spheroidal) of oscillations of relativistic magnetized stars has been calculated. Analytic solutions of Maxwell's equations in the internal and external background space-time of a slowly rotating misaligned magnetized neutron star have been obtained. A formula for magneto-dipolar radiation of rotating stars derived in general theory gives a correction for electromagnetic energy loss of $2-6$ times, depending on the compactness of the neutron star.

In a cosmological setting, the theory of macroscopic gravity is a large-distance scale generalization of general relativity. The averaged Einstein equations of macroscopic gravity are modified by the gravitational correlation tensor terms as compared with the Einstein equations of general relativity, the tensor satisfying an additional system of equations. Exact cosmological solutions to the equations of macroscopic gravity for a flat $(k=0)$ spatially homogeneous, isotropic space-time are found. The gravitational correlation terms in the averaged Einstein equations have the form of spatial curvature, dark matter and dark energy (cosmological constant) with particular equations of state for each correlation regime. The interpretation of these cosmological models to explain the observed large-scale structure of the accelerating Universe with a significant amount of the nonluminous (dark) matter is discussed.

In the following two sections we will provide examples of theoretical research carried out in our groups.

\section{Collapsing models of early evolution stages of self-gravitating systems: non-linear spherical and disk-like models}

One of the urgent problems of present-day astrophysics, the galactic and extra-galactic astronomy, is to study the early non-stationary evolution stages and development processes of galaxy large-scale structure (Binney \& Merrifield 1998) of galaxies and other self-gravitating systems. This problem calls for complex numerical calculations with modern computers, or constructing non-linear non-stationary models as well as studying the problem of their stability. The second method seems more preferable, as at the numerical experiments it is difficult to reveal some non-linear effects and to take notice of gravitational instabilities that originate in proper time.

The non-stationary phase-pulsating models, which are adequate for the investigation of the instabilities of early evolution stages of galaxies and their superclusters, have been constructed (Nuritdinov 1983, Nuritdinov 1991). In particular, we obtained the models that generalize the Einstein and Kamm equilibrium models for spherical self-gravitating systems in the non-stationary case.

The phase density of spherical self-gravitating system in the generalized Einstein model is

$\Psi_{1}\left(r, \mathrm{v}_{\mathrm{r}}, \mathrm{v}_{\perp}, \mathrm{t}\right)=\rho(\mathrm{t})\left[2 \pi \mathrm{v}_{\mathrm{b}}(\mathrm{t})\right]^{-1} \delta\left(\mathrm{v}_{\mathrm{r}}-\mathrm{v}_{\mathrm{a}}\right) \delta\left(\mathrm{v}_{\perp}-\mathrm{v}_{\mathrm{b}}\right) \chi\left(\mathrm{R}_{0} \Pi-\mathrm{r}\right)\left[1+\Omega \frac{\mathrm{v}_{\perp}}{\mathrm{v}_{\mathrm{b}}} \sin \theta \sin \eta\right]$.

The phase density of spherical self-gravitating system in the generalized Kamm's model is

$$
\Psi_{2}\left(r, \mathrm{v}_{\mathrm{r}}, \mathrm{v}_{\perp}, \mathrm{t}\right)=\rho \Pi^{4} /\left(\pi^{2} \mathrm{R}^{2} \Omega_{0}^{2}\right) \cdot \mathrm{f}^{-1 / 2} \cdot \chi(\mathrm{f})\left[1+\Omega \frac{\mathrm{r} \cdot \mathrm{v}_{\mathrm{r}}}{\Omega_{0} \mathrm{R}_{0}^{2}} \sin \theta \sin \eta\right],
$$


where variables are

$$
\mathrm{f}=\left(1-\mathrm{r}^{2} / \mathrm{R}^{2}\right) \cdot\left(\Omega_{0}^{2} \mathrm{R}^{2} / \Pi^{4}-\mathrm{v}_{\perp}^{2}\right)-\left(\mathrm{v}_{\mathrm{r}}-\mathrm{v}_{\mathrm{a}}\right)^{2}, \quad \mathrm{v}_{\mathrm{a}}=\frac{\Omega_{0} \lambda \mathrm{r} \sin \psi}{\sqrt{1-\lambda^{2}} \Pi^{2}}, \quad \mathrm{v}_{\mathrm{b}}=\frac{\mathrm{r} \cdot \Omega_{0}}{\Pi^{2}},
$$

and

$$
\Pi(\psi)=\frac{1+\lambda \cos \psi}{1-\lambda^{2}}, \quad \sin \theta=\frac{\sqrt{\mathrm{x}^{2}+\mathrm{y}^{2}}}{\mathrm{r}}, \quad \eta=\arctan \left(\frac{\mathrm{v}_{\varphi}}{\mathrm{v}_{\theta}}\right) .
$$

Here $\rho(t)$ is the matter density in physical space and it does not depend on $r ; \delta$ and $\chi$ are the symbols of Dirac and Heaviside functions; $\mathrm{v}_{\varphi}$ and $\mathrm{v}_{\theta}$ are the azimuthal and meridian components of velocity $\mathrm{v}_{\perp} ; \Pi(\psi)$ is the expansion factor of sphere; the variable $\psi$ is connected with $t$ by correlation $\Omega_{0} t=\left(1-\lambda^{2}\right)^{-3 / 2}(\psi+\lambda \sin \psi) ; \Omega_{0}$ is the frequency of star revolution in its orbit of the stationary system; $\lambda=1-(2 T /|U|)_{0}$ is the pulsation amplitude, where $(2 T /|U|)_{0}$ is the virial ratio with $t=0, T$ and $U$ being the system kinetic and potential energies, respectively. $\Omega$ is the angular velocity of the system rotation.

Real evolution of collapsing galaxies is described by the function $\Psi+\Delta \Psi_{i}$ which should satisfy the system of Jeans-Poisson equations. The above-mentioned models are applicable to early evolution stages of elliptical $E$ and lens-like $S$ galaxies. For galaxy superclusters which are Zeldovich pancakes, the construction of disk-like non-linear nonequilibrium models is required. In this case the isotropic model for phase density

$$
\Psi_{\text {Is }}=\frac{\sigma_{0}}{2 \pi \Pi \sqrt{1-\Omega^{2}}}\left[\frac{1-\Omega^{2}}{\Pi^{2}}\left(1-\frac{r^{2}}{\Pi^{2}}\right)-\left(\mathrm{v}_{\mathrm{r}}-\mathrm{v}_{\mathrm{a}}\right)^{2}-\left(\mathrm{v}_{\perp}-\mathrm{v}_{\mathrm{b}}\right)^{2}\right]^{-1 / 2}
$$

is constructed.

This model is non-stationary generalization of the Zeldovich-Bisnovatiy-Kogan equilibrium disk and on this basis a number of new non-stationary models for early evolution stage of self-gravitating disk-like systems with the anisotropic velocity diagrams (Nuritdinov 1992, Mirtadjieva, 2003) is constructed:

$$
\Psi_{\mathrm{A}}=\int_{-1}^{+1} \Psi_{I s} A\left(\Omega^{\prime}\right) d \Omega^{\prime}
$$

For $A\left(\Omega^{\prime}\right)=(2 / \pi)\left(1-\Omega^{\prime 2}\right)^{1 / 2}\left(1+\Omega \cdot \Omega^{\prime}\right)$

$$
\Psi_{A}^{1}=\frac{\sigma_{0}}{\pi}\left(1+\Omega^{\prime} \frac{\left[\mathrm{xv}_{\mathrm{y}}-\mathrm{yv}_{\mathrm{x}}\right]}{R_{0}^{2}}\right) \chi(D)
$$

For $A\left(\Omega^{\prime}\right)=1 / 2$

$$
\Psi_{A}^{2}=\alpha \cdot F(\beta, k)
$$

where $F(\beta, k)$ is spatial function in the form of the Jacobi elliptical integral.

$$
\text { For } \begin{aligned}
& A\left(\Omega^{\prime}\right)=(8 / 3 \pi) \cdot\left(1-\Omega^{\prime 2}\right)^{3 / 2} \\
& \qquad \Psi_{A}^{3}=\frac{2 \sigma_{0}}{3 \pi}\left\{1+\frac{r^{2}}{\Pi^{2}}+\Pi^{2}\left[\left(\mathrm{v}_{\mathrm{r}}-\mathrm{v}_{\mathrm{a}}\right)^{2}+\mathrm{v}_{\perp}^{2}\right]-3 \mathrm{r}^{2} \mathrm{v}_{\perp}^{2}\right\} \chi(D) .
\end{aligned}
$$

For $A\left(\Omega^{\prime}\right)=\Omega^{\prime 4} \cdot\left(1-\Omega^{\prime 2}\right)^{1 / 2}$

$$
\Psi_{A}^{4}=\frac{\sigma_{0}}{\pi}\left(3 D^{2}+24 D \cdot \mathrm{r}^{2} \mathrm{v}_{\perp}^{2}+8 \mathrm{r}^{4} \mathrm{v}_{\perp}^{4}\right) \chi(D) .
$$


The method of constructing non-linear models is based on the linear superposition of any two above-stated models. By this way we can obtain a new anisotropic model with the compound nature since their gravitational potentials and surface densities coincide:

$$
\Psi\left(\overrightarrow{\mathrm{r}}, \overrightarrow{\mathrm{v}}, \Omega_{1}, \Omega_{2}, \lambda, \nu, \mathrm{t}\right)=(1-\nu) \cdot \Psi_{\mathrm{i}}\left(\overrightarrow{\mathrm{r}}, \overrightarrow{\mathrm{v}}, \Omega_{1}, \nu, \mathrm{t}\right)+\nu \cdot \Psi_{\mathrm{j}}\left(\overrightarrow{\mathrm{r}}, \overrightarrow{\mathrm{v}}, \Omega_{2}, \nu, \mathrm{t}\right)
$$

here $\nu$ is the parameter of superposition.

This method allows us to construct the multiple parameter models which describe conditions of systems being close to real existing early stages of its evolution. On the other hand, by this way we construct a model which covers intermediate conditions between two discrete non-stationary configurations.

\section{Averaging problem in cosmology and macroscopic gravity}

Macroscopic gravity is a non-perturbative geometrical approach proposed in Zalaletdinov (1992) - Zalaletdinov (2003) to resolve the averaging problem of general relativity by its reformulation in a broader context as the problem of the macroscopic description of gravitation. The classical physical phenomena are known to possess two levels of description: the microscopic description by discrete matter models and the macroscopic description by the continuous matter models. Transition from a microscopic picture to a macroscopic one is accomplished by means of a space-time or ensemble averaging procedure. Lorentz' theory of electrons and Maxwell's electrodynamics are known examples of a microscopic theory and its macroscopic theory

$$
\begin{aligned}
F_{, \nu}^{\mu \nu}=\frac{4 \pi}{c} j^{\mu}=4 \pi \sum_{i} q_{i} u^{\mu}\left(t_{i}\right) & \rightarrow\langle\operatorname{avg}\rangle \rightarrow \quad H^{\mu \nu}{ }_{, \nu}=\frac{4 \pi}{c}\langle j\rangle^{\mu}=\frac{4 \pi}{c}\left(J^{\mu}-c P_{, \nu}^{\mu \nu}\right) \\
F_{[\alpha \beta, \gamma]}=0 & \rightarrow\langle\operatorname{avg}\rangle \rightarrow\langle F\rangle_{[\alpha \beta, \gamma]}=0, \quad H^{\mu \nu}=\langle F\rangle^{\mu \nu}+4 \pi P^{\mu \nu}
\end{aligned}
$$

where avg is averaging.

For general relativity, such a task is much more complicated because of (1) the necessity to define covariant space, space-time volume or statistical averages on Riemannian space-times, (2) the Riemannian geometry of space-time, the nonlinear structure of the field operator of the Einstein equations and necessity to deal with gravitational field correlators, and (3) the problem of construction of models of smoothed, continuously distributed self-gravitating media $T_{\alpha \beta}^{(\text {hydro })}=\left\langle T_{\alpha \beta}^{(\text {micro })}\right\rangle$. The Einstein equations themselves can be shown to be insufficient to be consistently averaged out.

$$
\begin{aligned}
\left\langle R_{\alpha \beta}\right\rangle-\frac{1}{2}\left\langle g_{\alpha \beta} g^{\mu \nu} R_{\mu \nu}\right\rangle & =-\kappa\left\langle T_{\alpha \beta}^{(\text {micro })}\right\rangle \Rightarrow\left\langle R_{\alpha \beta}\right\rangle-\frac{1}{2}\left\langle g_{\alpha \beta}\right\rangle\left\langle g^{\mu \nu}\right\rangle\left\langle R_{\mu \nu}\right\rangle+C_{\alpha \beta} \\
& =-\kappa\left\langle T_{\alpha \beta}^{(\text {micro })}\right\rangle .
\end{aligned}
$$

They become a definition of the correlation function $C_{\alpha \beta}$ unless this object is defined from outside the averaged Einstein equations. In macroscopic gravity provides a formalism for a derivation of the averaged Einstein's equations which take the form

$$
\bar{g}^{\alpha \epsilon} M_{\epsilon \beta}-\frac{1}{2} \delta_{\beta}^{\alpha} \bar{g}^{\mu \nu} M_{\mu \nu}=-\kappa\left\langle T_{\beta}^{\alpha(\text { micro })}\right\rangle+\left(Z_{\mu \nu \beta}^{\alpha}-\frac{1}{2} \delta_{\beta}^{\alpha} Q_{\mu \nu}\right) \bar{g}^{\mu \nu}
$$

where $\bar{g}^{\mu \nu}$ is the averaged metric, $M_{\mu \nu}$ is the Ricci tensor of the Riemannian curvature $M^{\alpha}{ }_{\beta \gamma \delta}$ which stands for the induction tensor, $Z^{\alpha}{ }_{\mu \nu \beta}-\frac{1}{2} \delta_{\beta}^{\alpha} Q_{\mu \nu}$ is a correlation tensor constructed from the correlation connection tensor,

$$
Z_{\beta \gamma}^{\alpha}{ }_{\nu \sigma}^{\mu} \equiv Z_{\beta\left[\gamma^{\mu} \sigma\right]}^{\alpha}=\left\langle\Gamma_{\beta[\gamma}^{\alpha} \Gamma_{\underline{\nu} \sigma]}^{\mu}\right\rangle-\left\langle\Gamma_{\beta[\gamma}^{\alpha}\right\rangle\left\langle\Gamma^{\mu}{ }_{\underline{\nu} \sigma]}\right\rangle
$$


where angular brackets denote a space-time volume averaging (Zalaletdinov (1992) Zalaletdinov (1993)). There correlation tensors and another non-Riemannian curvature tensor $R_{\beta \gamma \delta}^{\alpha}$ which stand for polarization tensors and average field tensors satisfy a set of non-linear partial differential equations.

An exact cosmological solution to the equations of macroscopic gravity has been obtained by Coley, Pelavas \& Zalaletdinov (2005) for a flat spatially homogeneous, isotropic macroscopic space-time given by the Robertson-Walker line element

$$
d s^{2}=-d t^{2}+a^{2}(t)\left(d x^{2}+d y^{2}+d z^{2}\right)
$$

for the constant macroscopic gravitational correlation tensor

$$
Z^{\alpha}{ }_{\beta \gamma}{ }^{\mu}{ }_{\nu \sigma}=\mathrm{const}
$$

which means that the macroscopic gravitational correlations are assumed to remain unchanged in time and space. The macroscopic gravity equations can be solved to show that there is finally only one remaining independent component $Z^{3}{ }_{23}{ }^{3}{ }_{32}$ determined through an integration constant.

The equation of state for the macroscopic gravitational correlation field

$$
\left.p_{\text {grav }}=\operatorname{pgrav}_{\operatorname{ggrav}}\right)=-\frac{1}{3} \rho_{\text {grav }}
$$

is determined by the equations of macroscopic gravity and the averaged Einstein equations with an averaged matter distribution taken to be a perfect fluid read

$$
\begin{aligned}
\left(\frac{\dot{a}}{a}\right)^{2} & =\frac{\kappa \rho_{\text {mat }}}{3}+\frac{\varepsilon}{3 a^{2}}, \\
2 \frac{\ddot{a}}{a}+\left(\frac{\dot{a}}{a}\right)^{2} & =-\kappa p_{m a t}+\frac{\varepsilon}{3 a^{2}},
\end{aligned}
$$

with a given equation of state $p_{\text {mat }}=\left(\rho_{\text {mat }}\right)$, where $\varepsilon / \kappa a^{2}=\rho g r a v$ is the macroscopic gravitational correlation energy density and $-\varepsilon / 3 \kappa a^{2}=p_{\text {grav }}$ is the isotropic pressure of macroscopic gravitational correlation field. They look similar to the Einstein equations of general relativity for either a closed or an open spatially homogeneous, isotropic Friedman-Lemaitre-Robertson-Walker space-time, but they do have different mathematical and physical, and therefore, cosmological content since

$$
\frac{\varepsilon}{3}=\frac{\kappa \rho g r a v a^{2}}{3} \neq-k
$$

in general due to the presence of macroscopic gravitational correlation terms of a spatial curvature term $\varepsilon / 3 a^{2}$. This macroscopic gravitational correlation terms is a candidate for a "dark" cosmological agent with the following physical properties.

\section{Conclusions}

We may conclude for the results presented in sections 2 and 3. Early stages of galaxy formation and its large scale structures are investigated by studying instabilities of the models specified with respect to structural perturbations (Nuritdinov et al. 1997, Nuritdinov et al. 2003).

A criterion of elliptical galaxy formation is found which reads that the initial total kinetic energy of a non-rotating system must not exceed $4,2 \%$ of the total initial potential 
energy. In this process the Jeans instability mechanism for almost radial motion acts. Besides, the process of elliptical galaxy formation goes in the same way if the percentage specified lies in the interval $(7-19 \%)$, but in this case another instability mechanism acts which has an oscillatory and resonance character. When the rotation effect is included, the percentage mentioned above grows.

A new kind of instability is discovered for pulsating spherical and disk-like galaxies, which has a combinatory-resonance character.

It is found for the first time that, among the modes studied, the increment of instability of pear-shaped perturbations is apparently larger than that of all the remaining modes, including the bar mode.

A formation criterion is found also for SB galaxies: the initial value of the total kinetic energy must not exceed $10,4 \%$ of the initial potential energy of a non-rotating system.

Instabilities of small scale modes are studied and their applications are specified.

Thus, the physics of instability of non-linear non-equilibrium models, which describe the early stages of the evolution of galaxies and their systems, differs significantly from that of the corresponding equilibrium configurations.

The dark spatial curvature term has the following physical properties as a cosmological agent:

(A) it interacts only gravitationally with the macroscopic gravitational field,

(B) it does not interact directly with the energy-momentum tensor of matter,

(C) it exhibits a negative pressure $p_{\text {grav }}=-\frac{1}{3} \rho$ grav which tends to accelerate the Universe when $\rho$ grav $>0$.

Only if one requires $12 Z^{3}{ }_{23}{ }^{3} 32=-\varepsilon$ to be

$$
\varepsilon=-3 k
$$

then the macroscopic (averaged) Einstein equations become exactly the Einstein equations of general relativity for either a closed or an open spatially homogeneous, isotropic space-time for the macroscopic geometry of a flat spatially homogeneous, isotropic spacetime.

This exact solution of the macroscopic gravity equations exhibits a very non-trivial phenomenon from the point of view of the general-relativistic cosmology: the macroscopic (averaged) cosmological evolution in a flat Universe is governed by the dynamical evolution equations for either a closed or an open Universe depending on the sign of the macroscopic energy density $\rho$ grav with a dark spatial curvature term $\kappa \rho$ grav $/ 3$. From the observational point of view such a cosmological model provides a possibility to formulate a new paradigm to reconsider the standard cosmological interpretation and treatment of the observational data. Indeed, this macroscopic cosmological model has the geometry of a flat spatially homogeneous, isotropic space-time. Therefore, all measurements and data are to be considered and designed for this geometry. The dynamical interpretation of the data obtained should be considered and treated for the cosmological evolution of either a closed or an open spatially homogeneous, isotropic space-time.

\section{Acknowledgements}

This research is supported in part by the UzFFR (project 01-06) and projects F2.1.09, F2.2.01, F2.2.06 and A13-226 of the UzCST. BJA acknowledges the partial financial support from NATO through the reintegration grant EAP.RIG.981259. RMZ acknowledges the partial financial support by the RCD Grant from NSERC of Canada and the W.F. James Chair research grant at St Francis Xavier University. 


\section{References}

Binney, J., Merrifild, M. 1998, Galactic Astronomy, Princeton University Press, 1998

Nuritdinov, S.N. , 1983 Sov. Astronomy, v. 27, p. 24

Nuritdinov, S.N., 1991 Sov. Astronomy, v. 35, p. 377

Nuritdinov, S.N., 1992 Astron. Tsir., N. 1553, p. 9

Nuritdinov, S.N., Gaynullina, E.R., Mirtadajieva, K.T. , 1997 Central regions of the Galaxy and gaalxies, IAU Symposium 184, Kyoto, p. 49

Nuritdinov, S.N., Mirtadajieva, K.T., Tadjibaev, I.U. ,2003 ASPS, v. 316, p. 377

Mirtadajieva, K.T. ,2003 Uzbek Journal of Physics., v. 5, p. 223

R.M. Zalaletdinov, Gen. Rel. Grav. 24 (1992) 1015.

R.M. Zalaletdinov, Gen. Rel. Grav. 25 (1993) 673.

R.M. Zalaletdinov, Bull. Astr. Soc. India 25 (1997) 401; gr-qc/9703016, 11 p.

R.M. Zalaletdinov, Ann. European Acad. Sci. (2003) 344.

A.A. Coley, N. Pelavas, and R.M. Zalaletdinov, Phys. Rev. Lett. 95 (2005) 151102.

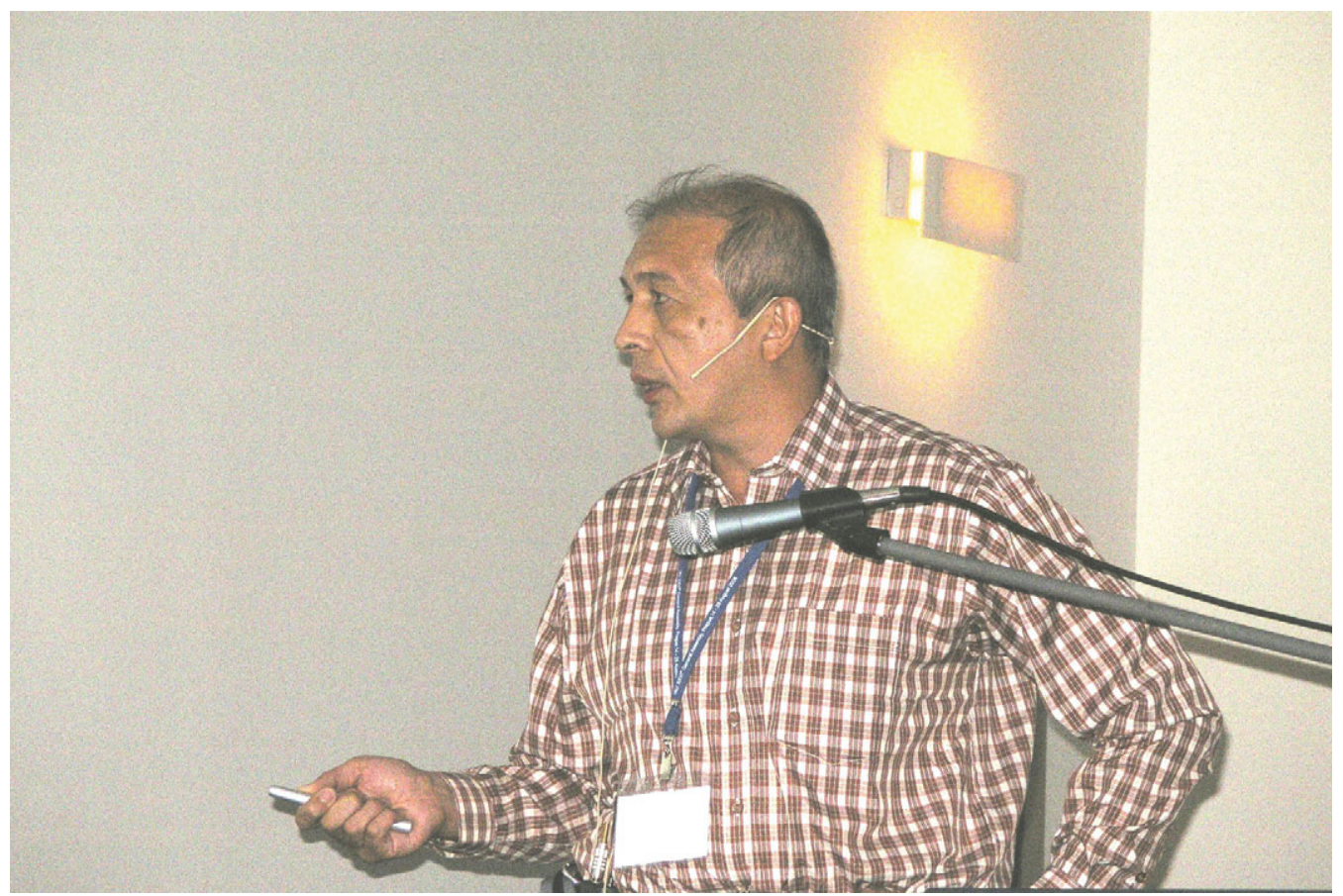

Bobomurat Ahmedov 